\title{
Lymphocyte-activation Gene 3 and Programmed Cell Death 1 Checkpoint Inhibition as a Novel Combination in Advanced Melanoma
}

\author{
Michael Serzan ${ }^{1-3}$ and Michael B Atkins ${ }^{1-3}$ \\ 1. Lombardi Comprehensive Cancer Center, Washington, DC, USA; 2. Georgetown University Medical Center, Washington, DC, USA; \\ 3. MedStar Georgetown University Hospital, Washington, DC, USA
}

DOI: https://doi.org/10.17925/OHR.2021.17.2.58

mmune checkpoint inhibition with programmed cell death 1 (PD-1) and cytotoxic T-lymphocyte-associated antigen 4 blockade has revolutionized the treatment of patients with advanced melanoma, leading to unprecedented outcomes. However, many trials have targeted additional co-inhibitory or co-stimulatory immune checkpoints with limited success. RELATIVITY-047 is a phase II/III trial of a novel combination involving relatlimab (anti-lymphocyte-activation gene 3 [LAG-3]) plus nivolumab (anti-PD-1), which reported an early progressionfree survival benefit with a favourable adverse effect profile. We discuss the key implications and unresolved questions regarding the role of dual LAG-3/PD-1 checkpoint blockade in advanced melanoma.

\section{Keywords}

Relatlimab, nivolumab, LAG-3 inhibition, PD-1 inhibition, immunotherapy, melanoma

Disclosures: Michael B Atkins serves on advisory boards for BMS, Merck, Novartis, Pfizer, Genentech-Roche, Exelixis and Eisai; and is a consultant for Agenus, AstraZeneca, IOVANCE, Pyxis Oncology, and Werewolf. Michael Serzan has no financial or non-financial relationships or activities to declare in relation to this article.

Review process: Double-blind peer review. Compliance with ethics: This article is an opinion piece and does not report on new clinical data, or include any studies with human or animal subjects performed by any of the authors.

Authorship: The named authors meet the International Committee of Medical Journal Editors (ICMJE) criteria for authorship of this manuscript, take responsibility for the integrity of the work as a whole, and have given final approval for the version to be published.

Access: This article is freely accessible at touchONCOLOGY.com (c) Touch Medical Media 2021

Received: 26 July 2021

Accepted: 2 September 2021

Published online: 9 November 2021

Citation: touchREVIEWS in Oncology \& Haematology 2021;17(2):58-9

Corresponding author: Michael B Atkins, William M Scholl Professor of Medicine, Department of Oncology, Georgetown Lombardi Comprehensive Cancer Center, 3970 Reservoir Rd, NW Research Building, Room E501, Washington, DC 20057, USA. E: mba41@georgetown.edu

Support: No funding was received for the publication of this article.
RELATIVITY-047 reported on the initial efficacy and safety of relatlimab (RELA), an antibody to lymphocyte-activating gene 3 (LAG-3), in combination with nivolumab (NIVO), an antibody to programmed cell death 1 (PD-1). ${ }^{1}$ These positive findings add to the existing evidence for the efficacy of anti-PD-1 therapy - either alone or in combination with ipilimumab (IPI), an antibody to cytotoxic T-lymphocyte-associated antigen 4 - for patients with advanced melanoma. In the CheckMate 067 trial, combination NIVO and IPI demonstrated plateaus in progression-free survival (PFS) (34\%) and overall survival (OS) (49\%), and a median treatment-free interval of 27.6 months at a minimum 6.5 years' follow-up. ${ }^{2}$ NIVO plus IPI also produced considerable toxicity, with grade 3-4 treatment-related adverse events (TRAEs) in 59\% of patients. Phase III studies of anti-PD-1 monotherapy with NIVO or pembrolizumab (PEMBRO) have demonstrated plateaus in PFS (23-28\%) and OS (38-39\%) at median follow-up of 5 years. ${ }^{3.4}$ NIVO or PEMBRO monotherapy was better tolerated than combination NIVO/IPI, with grade 3-4 TRAEs in 16-17\% of patients. Despite these promising results, many patients exhibit primary or acquired resistance to anti-PD-1-based therapy or experience immune-mediated adverse events that limit its use.

LAG-3 is a transmembrane protein expressed on activated $C D 4^{+}$and $C D 8^{+} T$ cells, where it acts as a competitive ligand for major histocompatibility complex II, leading to an exhausted T-cell phenotype. ${ }^{5}$ Preclinical studies have demonstrated that concurrent blockade of LAG-3 and the PD-1 pathway reinvigorates antitumour immunity through reactivating CD8+ effector $\mathrm{T}$ cells and upregulating inflammatory cytokines. ${ }^{.}$A phase $1 / /$ ll study in patients with advanced melanoma who progressed on anti-PD-1/programmed death-ligand 1 (PD-L1) therapy demonstrated the combination of RELA with NIVO had an objective response rate (ORR) of $16 \%$ and a disease control rate of $45 \%$, with grade $3-4$ TRAEs occurring in only $9 \%$ of patients.?

RELATIVITY-047 is a phase II/III trial for patients with treatment-naïve, unresectable or metastatic melanoma randomized to RELA 160 mg plus NIVO 480 mg, or NIVO 480 mg, given every 4 weeks. ${ }^{1}$ The study met its primary outcome with RELA plus NIVO demonstrating significant improvement in median PFS compared with NIVO monotherapy: 10.1 versus 4.6 months, respectively (hazard ratio [HR] 0.75, 95\% confidence interval [CI] 0.6-0.9]; $p=0.0055$ ). Subgroup analysis demonstrated benefit regardless of BRAF mutation status, American Joint Committee on Cancer stage or LAG-3 expression. Interestingly, patients with tumour cell PD-L1 expression $<1 \%$ showed greater PFS benefit from RELA plus NIVO than with NIVO alone (HR 0.66, 95\% Cl 0.51-0.84). The combination was associated with higher rates of grade $3-4$ TRAES than NIVO monotherapy (18.9\% versus $9.7 \%$, respectively). OS results were immature and ORR remained blinded at the time of presentation in accordance with the study's planned hierarchical outcome assessment.

While these early outcomes demonstrate efficacy of RELA plus NIVO, several unanswered questions remain. First, RELATIVITY-047 was designed as a gated phase II/III study, resulting in follow-up ranging widely, from 1.3 to 33.1 months. As data continue to mature, particular interest 
will be given to whether the PFS benefit for RELA plus NIVO persists and extends to an OS benefit. Second, RELA and NIVO were administered as fixed-dose infusions given without interruption at 4-week intervals until progression or toxicity. Longer-term efficacy, toxicity and quality of life outcomes are necessary to understand how this combination, with its more protracted administration schedule, compares with induction NIVO plus IPI for up to 12 weeks followed by NIVO maintenance and, further, whether durable tumour response is possible without the need for ongoing treatment. ${ }^{8}$

RELA plus NIVO is an active regimen that will likely be used in the metastatic, neoadjuvant and adjuvant settings. In the neoadjuvant setting, RELA plus NIVO prompted pathological complete responses in $57 \%$ of patients with regional disease, a major pathological response rate of $66 \%$, and no disease relapses in these responding patients at 16 months, thereby supporting the antitumour efficacy of this combination. ${ }^{9}$ No grade $3-4$ adverse effects occurred prior to surgery, and occurred in $26 \%$ of patients on subsequent adjuvant RELA plus NIVO therapy. However, despite the impressive efficacy of RELA plus NIVO, based on the data to date NIVO plus IPI will likely remain the standard of care for patients with a BRAF mutation, high lactate dehydrogenase
$(\mathrm{LDH})$, or central nervous system or liver metastases, given the strong clinical data supporting this regimen in these patient subsets. ${ }^{10}$ Further, there may be an emerging role for reverse ratio NIVO $3 \mathrm{mg} / \mathrm{kg}$ plus IPI $1 \mathrm{mg} / \mathrm{kg}$, which has demonstrated a significantly lower incidence of grade 3-5 TRAEs with no differences in efficacy endpoints compared with NIVO $1 \mathrm{mg} / \mathrm{kg}$ and IPI $3 \mathrm{mg} / \mathrm{kg}$ in some of these patient subsets. For patients without a BRAF mutation, normal LDH, or M1a/b disease, RELA plus NIVO may be favoured over NIVO monotherapy, particularly in those with tumour PD-L1 expression $<1 \%$. However, the current data suggest that anti-PD-1 monotherapy may be as efficacious as RELA plus NIVO for patients with tumour PD-L1 expression $>1 \%$. Given the heavy use of anti-PD-1 monotherapy in the adjuvant setting, studies of RELA plus NIVO for high-risk melanoma are particularly appealing, and are in the planning stages (NCT05002569). ${ }^{11}$

RELATIVITY-047 provides evidence supporting RELA plus NIVO as a breakthrough novel immunotherapeutic combination, producing a PFS benefit with adverse effect profile more favourable than NIVO plus IPI. Further studies investigating the ability of LAG-3 and PD-1 inhibition to reinvigorate exhausted $T$ cells should be done in other advanced malignancies. •
1. Lipson EJ, Tawbi HA, Schadendorf D, et al. Relatlimab (RELA) plus nivolumab (NIVO) versus NIVO in first-line advanced melanoma: primary phase III results from RELATIMTY 047 (CA224-047). J Clin Oncol. 2021;39(Suppl. 15):9503

2. Wolchok JD, Chiarion-Sileni V, Gonzalez $R$, et al. CheckMate 067 : 6.5-year outcomes in patients (pts) with advanced melanoma. J Clin Oncol 2021:39(Suppl. 15):9506.

3. Robert C, Long GV, Brady B, et al. Five-year outcomes with nivolumab in patients with wild-type BRAF advanced melanoma J Clin Oncol. 2020;38:3937-46.

4. Long GV, Schachter J, Arance A, et al. Long-term survival from pembrolizumab (pembro) completion and pembro retreatment: phase III KEYNOTE-006 in advanced melanoma. J Clin Oncol. 2020;38(Suppl. 15):10013.

5. Maruhashi T, Okazaki IM, Sugiura D, et al. LAG-3 inhibits the activation of $\mathrm{CD} 4+\mathrm{T}$ cells that recognize stable $\mathrm{pMHCIl}$ through its conformation-dependent recognition of pMHCll. Nat Immunol, 2018;19:1415-26.

6. Woo SR, Turnis ME, Goldberg MV, et al. Immune inhibitory molecules LAG-3 and PD-1 synergistically regulate T-cell function to promote tu-1 sy mistically regulate Tcell 2012;72:917-27.

7. Ascierto PA, Bono P, Bhatia S, et al. Efficacy of BMS-986016, a monoclonal antibody that targets lymphocyte activation gene-3 (LAG-3), in combination with nivolumab in pts with melanoma who progressed during prior anti-PD-1/PD-L1 therapy (mel prio IO) in all-comer and biomarker-enriched populations. Ann Oncol. 2017;28:v611-2.

8. Regan MM, Werner L, Rao S, et al. Treatment-free survival: a novel outcome measure of the effects of immune checkpoint inhibition - a pooled analysis of patients with advanced melanoma.J Clin Oncol. 2019;37:3350.
9. Amaria RN, Postow MA, Tetzlaff MT, et al. Neoadjuvant and adjuvant nivolumab (nivo) with anti-LAG3 antibody relatlimab (rela) for patients (pts) with resectable clinical stage III melanoma. I Clin Oncol 2021:39(Suppl. 15):9502.

10. Lebbé C, Meyer N, Mortier L, et al. Evaluation of two dosing ebbe $C$, Meyer, Morter $L$, et al. Evaluation of two dosing regimens for nivolumab in combination with ipilimumab in
patients with advanced melanoma: results from the phase $I \mathrm{Ib} / \mathrm{IV}$ patients with advanced melanoma: results from
CheckMate 511 trial. J Clin Oncol. 2019;37:867.

11. ClinicalTrials.gov. A study to assess adjuvant immunotherapy with relatlimab and nivolumab versus nivolumab alone after complete resection of stage III-IV melanoma (RELATIVITY-098). ClinicalTrials.gov Identifier: NCT05002569. Available at: https:// clinicaltrials.gov/ct2/show/NCT05002569 (accessed 12 October 2021) 